\section{HEART FAILURE}

\section{Study sheds light on importance of vitamin D in patients with HF}

In a study from Jerusalem, Israel, deficiency of vitamin $\mathrm{D}$ was both common and associated with increased mortality in patients with heart failure (HF). Vitamin D deficiency is highly prevalent around the world and is associated with multiple cardiovascular risk factors, such as diabetes mellitus, obesity, and hypertension.

Endogenous synthesis of vitamin D from exposure to sunlight is increasingly being limited by indoor lifestyles, even in sunrich countries like Israel, and particularly in patients whose activity levels are restricted by cardiovascular disease. Also, vitamin D is fat-soluble and can be sequestrated in the adipose tissue of individuals with obesity.

Investigators measured serum levels of 25-hydroxyvitamin $\mathrm{D}$ [25(OH)D] in 49,834 individuals $(n=3,009$ with $\mathrm{HF}$ and $n=46,825$ controls) between January 2006 and June 2010. 25(OH)D levels $\geq 75 \mathrm{nmol} / \mathrm{l}$ and $<25 \mathrm{nmol} / \mathrm{l}$ are considered to be optimal and deficient, respectively. Patients with HF had lower 25(OH)D levels (36.9 nmol/l versus $40.7 \mathrm{nmol} / \mathrm{l})$, a higher prevalence of vitamin D deficiency (28\% versus $22 \%$ ), and a lower incidence of vitamin D sufficiency (8.8\% versus $10.1 \%$ ) than control individuals. A significant seasonal variation in $25(\mathrm{OH}) \mathrm{D}$ levels was observed in all participants, with peak levels recorded in June-August.

During follow-up (median 518 days), vitamin D deficiency was a predictor of increased mortality both in patients with HF (HR 1.52, 95\% CI 1.21-1.92, $P<0.001$ ) and controls (HR 1.91, 95\% CI 1.48-2.46, $P<0.00001)$. Vitamin D supplementation (800-1,000 units per day) was prescribed to $63 \%$ of patients with HF during follow-up, and was independently associated with a reduction in mortality (HR 0.68, 95\% CI 0.54-0.85, $P<0.0001)$. Calcium supplementation had no effect on outcome.

"This study depicts the magnitude of the problem of vitamin D deficiency in patients with HF, as well as in the general population, even in a country with

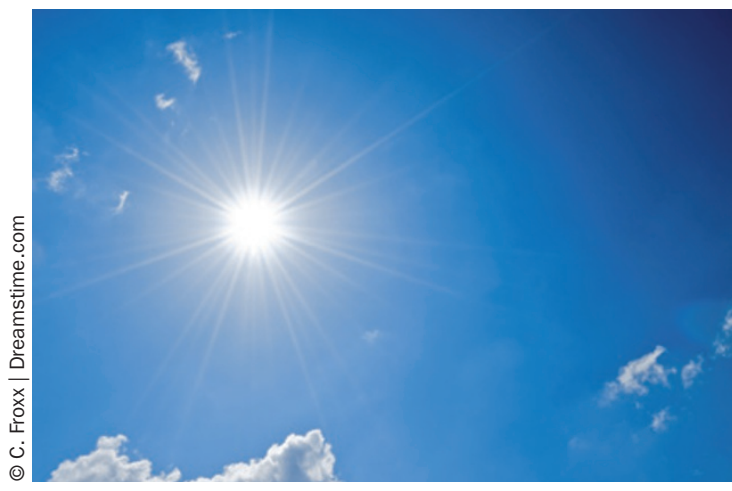

abundant solar radiation," comments lead author, Dr Israel Gotsman. Although these data indicate a "clear epidemiological relationship between vitamin D status and clinical outcome in [patients with] HF, and that vitamin $\mathrm{D}$ supplementation may influence outcome ... there is a definite need for further randomized controlled studies."

Gregory B. Lim

Original article Gotsman, I. et al. Vitamin D deficiency is a predictor of reduced survival in patients with heart failure; vitamin D supplementation improves outcome. Eur. J. Heart Fail. doi:10.1093/eurjhf/hfr175 